\title{
Efetividade de uma intervenção baseada em Mindfulness para redução de estresse e melhora da qualidade de vida em estudantes de enfermagem*
}

\author{
Edilaine Cristina da Silva Gherardi-Donato ${ }^{1,2}$ \\ (D) https://orcid.org/0000-0001-7475-6650 \\ Driele Cristina Gomes Quinhoneiro 3 \\ (D) https://orcid.org/0000-0003-2965-3999 \\ Larissa Bessani Hidalgo Gimenez ${ }^{2}$ \\ (D) https://orcid.org/0000-0002-3262-0023 \\ Leonardo Hernandes Siqueira ${ }^{2}$ \\ (D) https://orcid.org/0000-0001-6689-7832 \\ Kranya Victoria Diaz-Serrano 4,5 \\ (D) https://orcid.org/0000-0001-9497-0005 \\ Ana Carolina Guidorizzi Zanetti 6,2 \\ (D) https://orcid.org/0000-0003-0011-4510
}

Objetivo: avaliar a efetividade de uma intervenção para redução de estresse baseada em Mindfulness em aspectos relacionados à Qualidade de Vida, Atenção Plena e Estresse Percebido, em estudantes de graduação e pós-graduação em enfermagem. Método: aplicados instrumentos de avaliação para a construção de uma linha basal e posteriormente a amostra foi submetida a um Programa de Redução de Estresse e aumento da Qualidade de Vida baseado em Mindfulness, formatado em encontros semanais por oito semanas. Resultados: após a intervenção, houve diminuição do nível de Estresse Percebido, aumento do nível de Atenção Plena e melhora da Qualidade de Vida em âmbito psicológico. Conclusão: intervenções baseadas em Mindfulness se mostram efetivas e podem constituir um importante recurso para o gerenciamento do estresse e melhora na Qualidade de Vida dos estudantes.

Descritores: Atenção Plena; Saúde Mental; Qualidade de Vida; Estresse; Estudantes.

\section{Como citar este artigo}

Gherardi-Donato ECS, Quinhoneiro DCG, Gimenez LBH, Siqueira LH, Diaz-Serrano KV, Zanetti ACG. Mindfulnessbased intervention for nursing students: Effects on stress and quality of life. SMAD, Rev Eletrônica Saúde Mental Álcool Drog. 2020;16(3):33-43. doi: https://dx.doi.org/10.11606/issn.1806-6976.smad.2020.152589 


\title{
Mindfulness-based intervention for nursing students: Effects on stress and quality of life
}

\begin{abstract}
Objective: to evaluate the effectiveness of an intervention to reduce stress based on Mindfulness in aspects related to Quality of Life, Mindfulness and Perceived Stress in undergraduate and postgraduate students of Nursing. Method: evaluation instruments were applied to establish baseline data, and the sample was subsequently submitted to a Mindfulness Based Stress Reduction and Quality of Life, formatted eight consecutive weeks. Results: after the intervention, there was a decrease in the level of Perceived Stress, increase in the level of Mindfulness and improvement of the Quality of Life in the psychological area. Conclusion: interventions based in Mindfulness are effective and can offer important resources for managing stress and improving the Quality of Life of students.
\end{abstract}

Descriptors: Mindfulness; Mental Health; Quality of Life; Stress; Students.

\section{Efectividad de una intervencion basada en Mindfulness para reduccion de estres y mejora de la calidad de vida en estudiantes de enfermeria}

\begin{abstract}
Objetivo: evaluar la efectividad de una intervención para reducción de estrés basada en Mindfulness en relación a la Calidad de Vida, Atención Plena y Estrés Percibido, en universitarios y estudiantes de postgrado de Enfermería. Método: previamente se aplicaron instrumentos de evaluación para la construcción de una línea basal y posteriormente la muestra fue sometida a un Programa de Reducción de Estrés y aumento de la Calidad de Vida basado en Mindfulness, constituído de encuentros semanales, durante ocho semanas consecutivas. Resultados: se observó que después de la intervención, hubo disminución del nivel de Estrés Percibido, aumento del nivel de Atención Plena y mejora de la Calidad de Vida en ámbito psicológico. Conclusión: intervencones basadas en Mindfulness se muestran efectivas y pueden constituir un importante recurso para manejar el estrés y mejorar la Calidad de Vida de los estudiantes.
\end{abstract}

Descriptores: Atención Plena; Salud Mental; Calidad de Vida; Estrés; Estudiantes. 


\section{Introdução}

Os jovens que ingressam na Universidade encontram uma realidade muito diferente da qual estavam inseridos no Ensino Médio, tratando-se de um importante período de transição. Neste novo contexto, o universitário pode se deparar com um ambiente com altas demandas, pouco controle sobre as tarefas e o déficit ou ausência de apoio social da universidade. Resultante a isso, estudos na última década têm apontado que o meio acadêmico pode ser um ambiente com elevado potencial estressor(1-3).

Estudo recente realizado em instituição de ensino superior no Brasil, mostrou elevados níveis de estresse em estudantes, numa proporção de $50 \%{ }^{(4)}$. Mais especificamente na área da saúde, $87,9 \%$ dos estudantes dos últimos anos do curso de enfermagem apresentaram níveis moderados ou altos de estresse ${ }^{(5)}$, corroborando com outros estudos nacionais(6-7).

Esta situação não é restrita aos estudantes de graduação. Alunos de pós-graduação também seguem em condições estressantes ${ }^{(8)}$. Em estudo realizado em uma amostra de 2.157 estudantes de pós-gradução stricto sensu, brasileiros, que 46,8\% apresentavam níveis de estresse considerados altos ou muito altos, os quais estavam fortemente atrelados a fatores da vida acadêmica(9). $^{(9)}$.

O estresse é caracterizado por um estado em que o corpo aciona recursos fisiológicos para enfrentar uma ameaça externa ou interna ${ }^{(10)}$, desencadeando uma cascata de respostas neuroendócrinas que modulam diversas funções fisiológicas. No âmbito acadêmico, pode ser exemplificado como uma reação frente a apresentação de um trabalho, prova ou desempenho em estágio. A literatura revela que existe um limiar em que o estresse é natural e exerce efeito positivo na neuroplasticidade ${ }^{(11)}$. No entanto, quando o indivíduo não consegue superar a ameaça, seja pela intensidade do estressor ou por seu prolongamento, observa-se que manter este estado fisiológico alterado pode levar ao comprometimento de funções orgânicas ${ }^{(10,12)}$, como a depressão do estado imunológico e o aparecimento de psicopatologias ${ }^{(13-14)}$. Altos níveis de estresse e sua ocorrência crônica podem ter um impacto direto sobre a vida na universidade, sendo um consenso geral de que este é um dos principais fatores que levam o estudante a apresentar baixo rendimento acadêmico.

O estresse pode ser avaliado através de parâmetros fisiológicos, como marcadores inflamatórios e a dosagem de hormônios (principalmente o cortisol). Outra maneira de mensurar é a utilização de escalas psicométricas, como a Escala de Estresse Percebido (PSS) ${ }^{(15)}$, umas das mais empregadas para avaliar o estresse subjetivo.

O estresse crônico associa-se a outras aspectos da vida, como a Qualidade de Vida (QV), aspecto que pode ser impactado pelo contexto vivenciado pelo estudante na universidade. Nesse âmbito, dentre os fatores que contribuem para a diminuição da QV dos estudantes, podemos citar: sobrecarga de atividades, demanda de tempo integral e prazos curtos de tarefas, desgaste físico e mental, dores, desinteresse e irritação(16).

Nessa perspectiva, a baixa QV nos estudantes está diretamente relacionada a piora do desempenho e do sucesso do processo de ensino-aprendizagem, devido a capacidade diminuída de atenção, baixo nível de energia diária e sono ${ }^{(17)}$.

O contexto insalubre universitário pode levar os estudantes a adotarem estratégias de enfrentamento não saudáveis, como o abuso de álcool e tabaco(18). Nesse sentido, níveis significativos de estresse foram identificados em estudantes universitários de enfermagem, associados a uma alta prevalência de consumo dependente de álcool(19).

Averigua-se que o uso de substância varia de acordo com a área de conhecimento e característica do curso, nota-se que ansiolíticos e anfetamínicos, por exemplo, tiveram o dobro de prevalência de uso por estudantes de enfermagem quando comparado à estudantes de outros cursos $^{(18)}$. O que pode estar relacionado a uma baixa $Q V^{(20)}$.

Diante do contexto apresentado, a implementação de intervenções com propósito de redução de estresse e promoção de saúde mental se fazem necessárias no meio acadêmico(4). Nos últimos anos, as intervenções baseadas em Mindfulness, entendidas como práticas de sustentação atenção e da consciência ao momento presente, têm demonstrado resultados significativos e vêm sendo aplicadas em universidades norte-americanas ao redor do mundo, como a Duke University e a Brown University ${ }^{(21)}$. Uma metanálise com 24 estudos, envolvendo 1431 universitários da Inglaterra, Estados Unidos, Tasmania e Irã, evidenciou associações entre intervenções que utilizam Mindfulness e a diminuição de sintomas de ansiedade, níveis mais baixos de depressão e do hormônio cortisol, o principal hormônio relacionado ao estresse(22).

Mindfulness, termo traduzido para o português como "atenção plena" (AP), refere-se a um estado mental ou psicológico caracterizado pela regulação de forma intencional da atenção ao que está acontecendo agora. Trata-se de um estado de mente oposto à desatenção(23), com propósito no momento presente, de forma não julgadora, sendo este um recurso interno inerente a todos os seres humanos.

O cultivo de práticas de Mindfulness está correlacionado negativamente com ansiedade, depressão e estresse ${ }^{(24-26)}$. Ainda, conforme o indivíduo incorpora o cultivo da atenção plena nas atividades diárias, denominadas práticas informais, mais intensa é a redução do estresse decorrente da prática(21). 
Dentre as intervenções baseadas em Mindfulness mais utilizadas, estão a Terapia Cognitiva Baseada em Mindfulness (MBCT), Terapia Comportamental Baseada na Aceitação (ABBT) e o Programa de Redução de Estresse Baseado em Mindfulness (MBSR), sendo este último, o pioneiro e um dos mais populares pelos seus resultados positivos na população não clínica(21).

O MBSR, idealizado por Kabat-Zinn, é um programa estruturado de oito semanas, com encontros semanais de aproximadamente 2,5 horas e um período de retiro em silêncio. Estudos têm sido conduzidos para testar a eficácia do MSBR em diferentes contextos de saúde, como depressão, ansiedade, dor crônica e doenças relacionadas ao estresse(26-32). No que diz respeito à redução de estresse, especificamente em universitários, uma revisão recente analisou 15 estudos que utilizaram como intervenção o programa MBSR e encontrou que em $73 \%$ dos estudos analisados o protocolo foi eficaz para reduzir o estresse. Observou-se também que os resultados são duradouros, chegando a manter os efeitos positivos mesmo após um ano(33).

Mindfulness vem sendo estudado no Brasil nos últimos anos ${ }^{(34-38)}$, porém ainda existe uma lacuna significativa de estudos em contexto nacional, considerando as particularidades e realidade da população universitária e de pós-graduação brasileira.

Apesar dos diversos estudos evidenciando altos níveis de estresse e outros problemas psicológicos entre estudantes universitários(39-41), poucos estudos na literatura apontam intervenções eficazes para lidar com essa problemática. A partir dessa realidade, buscamos nesse estudo encontrar uma alternativa de promoção da saúde mental não farmacológica nessa população, para lidar assertivamente com os estressores pessoais e da vida acadêmica.

Esse estudo teve como objetivo avaliar os efeitos de uma intervenção baseada em Mindfulness nos níveis de AP, QV e EP em estudantes de graduação e pósgraduação em enfermagem.

\section{Método}

Trata-se de um estudo piloto, quase experimental, antes e depois, que visa a possibilidade de testar, avaliar, revisar e aprimorar as estratégias para o desenvolvimento de futuras pesquisas com intervenções baseadas em Práticas de Mindfulness.

Inicialmente foi realizada divulgação por meio de fixação de cartazes na Escola de Enfermagem de Ribeirão Preto, Universidade de São Paulo, assim como em redes sociais, sobre a realização de uma palestra introdutória sobre Mindfulness e Estresse, aberta a alunos de graduação e de pós-graduação, onde foram expostas a natureza, o foco e a estrutura do programa, bem como o compromisso exigido dos participantes durante o programa. Foram acolhidos os questionamentos e as dúvidas que os participantes em potencial possuíam sobre o programa.

Após a palestra, os sujeitos que demonstraram interesse em participar foram orientados a preencher dados de avaliação pré-programa, os quais consistiram de questionários sociodemográfico e de condições de saúde, de avaliação de Estresse Percebido, de avaliação da Atenção Plena e da Qualidade de Vida, e tomaram a decisão sobre a inscrição no Programa de Qualidade de Vida e Redução de Estresse Baseado em Mindfulness. A participação enquanto sujeito da pesquisa foi manifestada por meio da assinatura do Termo de Consentimento Livre e Esclarecido.

Foram definidos como critérios de inclusão ter 18 anos de idade ou mais, sem predileção pelo sexo ou raça, ser estudante do curso de graduação ou pósgraduação em enfermagem, na Universidade de São Paulo - USP, Campus de Ribeirão Preto. Foram excluídos aqueles sujeitos que relataram estar em fase aguda ou em tratamento de algum transtorno psiquiátrico como transtornos de humor, pensamento, personalidade ou abuso de substâncias.

Os participantes foram avaliados em dois momentos: antes do inicio da intervenção, com dados basais (T0) e após as oito semanas de intervenção (T1).

A coleta de dados ocorreu no Centro de Mindfulness e Terapias Integrativas da Escola de Enfermagem de Ribeirão Preto (EERP), situado no Campus de Ribeirão Preto da Universidade de São Paulo (USP).

Foram coletadas informações referentes à idade, sexo, grau de instrução, situação conjugal, número de filhos, religião, atividade física, hábito de fumar, uso de bebida alcoólica, número de horas de sono, perfil de morbidade, hábito intestinal, por meio de questionário sóciodemográfico.

Todos os participantes foram avaliados antes e ao término da intervenção em relação às seguintes variáveis: nível de AP, EP e QV.

Para avaliar o nível de Mindfulness, foi aplicada a Escala de Atenção e Consciência Plena (MAAS), traduzida e validada para o português, que consiste em um instrumento de auto relato, com 15 itens, com opções de resposta que variam de (1) quase sempre a (6) quase nunca. Esta escala foi adaptada e validada para o Brasil e trata-se de um instrumento unidimensional e fidedigno para a aplicação em estudantes universitários e adultos em geral(42). A MAAS avalia as frequências de estado de atenção de acordo com o tempo e todos os itens medem Mindfulness.

Para mensurar o nível de EP utilizamos a Escala de Estresse Percebido (PSS), traduzida e validada no Brasil(43). A PSS avalia, através de 14 itens, quão imprevisível, incontrolável e sobrecarregado os 
respondentes encontram suas vidas. As alternativas de resposta variam de zero a quatro $(0=$ nunca; $1=$ quase nunca; 2 = às vezes; $3=$ quase sempre; $4=$ sempre). 0 escore é obtido pela soma de todos os itens da escala; itens declarados positivamente $(4,5,6,7,9,10$ e 13) e marcados inversamente $(0=4,1=3,2=2,3=1$ e $4=0$ ). Os escores do PSS variam de 0 a 56 , com uma pontuação maior indicando mais estresse.

Para avaliar o nível da QV dos estudantes, foi utilizado o instrumento abreviado de avaliação da qualidade de vida (WHOQOL-BREF), desenvolvido a partir da WHOQOL-100, do Grupo de Qualidade de Vida da Organização Mundial da Saúde (OMS)(44). Este instrumento de domínio público é constituído de 26 perguntas, e as respostas seguem uma escala do tipo Likert de 1 a 5; quanto maior a pontuação melhor a QV. O WHOQOL-BREF avalia quatro domínios: físico, psicológico, relações sociais e meio ambiente. O estudo de validação demonstrou-se que a escala apresenta um bom desempenho e configura em uma alternativa útil e válida para mensurar a QV no Brasil(44).

Um total de 15 alunos, representando 2,8\% da população de estudantes na Escola de EERP/USP, deram início ao programa piloto, de forma voluntária. Ao longo do desenvolvimento da pesquisa cinco indivíduos desistiram, resultando num grupo de 10 estudantes ( $n=$ 10). Trata-se assim de uma amostra não probabilística, por conveniência.

Considerando que, de um modo geral, um Programa de Mindfulness congrega entre 10 e 15 pessoas, para melhor seguimento do mesmo, esse tamanho amostral foi apropriado para o desenvolvimento do projeto piloto.

A intervenção foi conduzida por uma instrutora certificada e qualificada para orientar práticas de Mindfulness e consistiu de sessões semanais ao longo de oito semanas, com duração de duas horas. A intervenção teve como objetivo o desenvolvimento de habilidades como: regulação atencional, regulação emocional, consciência corporal e mudança na perspectiva do self, com base no Programa de Redução de Estresse baseado em Mindfulness - MBSR ${ }^{(23)}$. Os temas das sessões foram descritas na Figura 1.

\begin{tabular}{|l|l|}
\hline Sessão Um & O que é Mindfulness: Saindo do Piloto Automático \\
\hline Sessão Dois & Mindfulness na Respiração \\
\hline Sessão Três & Mindfulness na Vida Diária \\
\hline Sessão Quatro & $\begin{array}{l}\text { Estendendo as habilidades de Mindfulness para } \\
\text { situações desafiadoras }\end{array}$ \\
\hline Sessão Cinco & Mindfulness, aceitação e ação habilidosa \\
\hline Sessão Seis & Dia do Silêncio \\
\hline Sessão Sete & Mindfulness e Compaixão \\
\hline Sessão Oito & Mindfulness para a Vida Toda \\
\hline
\end{tabular}

Figura 1 - Tema da Intervenção por Sessão do Programa Baseado em Mindfulness
Os dados foram analisados através do software estatístico Statistical Package for the Social Sciences ${ }^{\circledR}$ (SPSS). As características das variáveis estudadas foram submetidas à estatística descritiva, por meio de distribuição de frequências, números absolutos e percentuais, média, mínimo e máximo. Foram analisados nos constructos avaliados as diferenças de médias de amostra nos diversos tempos propostos no estudo por meio do teste $\mathrm{t}$ de Student com nível de significância de $5 \%$ e teste de Wilcoxon não paramétrico, com fins comparativos. Foi utilizado Pearson para testar a correlação entre as variáveis.

$\mathrm{O}$ estudo foi conduzido respeitando os preceitos éticos vigentes e dispostos na Resolução n466, de 12 de dezembro de 2012, do Conselho Nacional de Saúde. Aprovação do Comitê de Ética em Pesquisa sob CAAE: 77835617.1 .0000 .5393$.

\section{Resultados}

Dos 10 sujeitos que participaram na pesquisa, oito eram do sexo feminino $(80 \%)$ e dois do sexo masculino (20\%), seis alunos eram de graduação e quatro da pós-graduação. Desses, oito relataram possuir crença religiosa e seis afirmaram praticar essa crença regularmente. Quanto à idade, a média foi de 25,3 anos $(D P=4,0)$. Em relação aos dados coletados, cinco dos estudantes praticavam regularmente atividade física, nove referiram não fumar, seis referiram consumir bebidas alcoólicas, sendo de 1,5 a média da frequência de consumo semanal, sete negaram uso de medicamentos, e dos que relataram fazer uso, dois referiram uso de psicotrópicos. Quanto às horas de sono, a média foi de $7,1(\mathrm{DP}=1,0)$, sete relataram hábito intestinal regular ou de regular a ruim, sete negaram possuir doença crônica não transmissível e dos que relataram ter, referiram ser enxaqueca (Tabela 1).

Ao comparar as medidas obtidas pela aplicação das escalas MAAS, PSS e WHOQOL-BREF total e seus domínios, antes e após a intervenção baseada em Mindfulness, pelo Teste de Wilcoxon, observou-se diferença estatisticamente significante quando comparados os valores dos escores TO (antes da intervenção) e T1 (após a intervenção) resultantes das aplicações da MAAS, da PSS, assim como no domínio psicológico da WHOQOL-BREF. Depois da intervenção baseada em Mindfulness, os estudantes apresentaram maiores valores no nível de Mindfulness $(p=0,005)$ e no domínio psicológico relacionado à avaliação de QV $(p=0,017)$ e menores valores em relação ao EP $(p=0,037)$ (Tabela 2). 
Tabela 1 - Distribuição dos estudantes segundo as variáveis sociodemográficas e clínicas $(n=10)$. Ribeirão Preto, SP, Brasil, 2018

\begin{tabular}{|c|c|c|c|}
\hline Variável & n (\%) & $\begin{array}{l}\text { Média } \\
\left(\mathrm{DP}^{*}\right)\end{array}$ & $\begin{array}{c}\text { Mediana } \\
\left(\text { IC }^{\dagger}\right) \\
\end{array}$ \\
\hline \multicolumn{4}{|l|}{ Sexo } \\
\hline Feminino & $8(80)$ & & \\
\hline Masculino & $2(20)$ & & \\
\hline \multicolumn{4}{|l|}{ Crença religiosa } \\
\hline Sim & $8(80)$ & & \\
\hline Não & $2(20)$ & & \\
\hline \multicolumn{4}{|l|}{ Prática regular da crença religiosa } \\
\hline Sim & $6(60)$ & & \\
\hline Não & $2(20)$ & & \\
\hline Idade & & $25,3(4,0)$ & $25,0(20-30)$ \\
\hline \multicolumn{4}{|l|}{ Prática de atividade física } \\
\hline Sim & $5(50)$ & & \\
\hline Não & $5(50)$ & & \\
\hline \multicolumn{4}{|l|}{ Consumo de cigarro } \\
\hline Sim & $1(10)$ & & \\
\hline Não & $9(90)$ & & \\
\hline \multicolumn{4}{|l|}{ Consumo de bebidas alcoólicas } \\
\hline Sim & $6(60)$ & & \\
\hline Não & $4(40)$ & & \\
\hline Frequência semanal de consumo de bebidas alcoólicas & & $1,5(0,37)$ & $1,5(1)$ \\
\hline \multicolumn{4}{|l|}{ Uso de medicamentos } \\
\hline Sim & $3(30)$ & & \\
\hline Não & $7(70)$ & & \\
\hline \multicolumn{4}{|l|}{ Tipo de Medicamento } \\
\hline Psicotrópicos & $2(67)$ & & \\
\hline Outros & $1(33)$ & & \\
\hline Horas de sono por dia & & $7,1(1,0)$ & $7,2(4,5-8,0)$ \\
\hline \multicolumn{4}{|l|}{ Hábito intestinal } \\
\hline Regular/Regular a ruim & $7(70)$ & & \\
\hline Lento & $3(30)$ & & \\
\hline \multicolumn{4}{|l|}{$\mathrm{DCNT}^{\ddagger}$} \\
\hline Sim & $2(20)$ & & \\
\hline Não & $8(80)$ & & \\
\hline
\end{tabular}

*DP = Desvio Padrão; ${ }^{+}$IC $=$Intervalo de Confiança; ${ }^{\ddagger}$ DCNT = Doença Crônica Não Transmissível

Tabela 2 - Comparação dos escores das escalas MASS, PSS e WHOOQOL-BREF total e domínios, antes (T0) e após (T1) a intervenção baseada em Mindfulness. Ribeirão Preto, SP, Brasil, 2018

\begin{tabular}{|c|c|c|c|c|c|c|c|c|c|}
\hline \multirow{2}{*}{ Variáveis } & \multicolumn{4}{|c|}{ Antes (T0) } & \multicolumn{4}{|c|}{ Depois (T1) } & \multirow{2}{*}{ Valor $\mathrm{p}^{\prime}$} \\
\hline & Média & $\mathrm{DP}^{\dagger}$ & Mediana & (IC‡) & Média & $\left(\mathrm{DP}^{\dagger}\right)$ & Mediana & $\left(I C^{\ddagger}\right)$ & \\
\hline MAAS $\$$ & 45,8 & $(10,8)$ & 43,5 & $(29-68)$ & 57,4 & $(9,3)$ & 57,5 & $(45-75)$ & 0,005 \\
\hline PSS" & 28,8 & $(12,2)$ & 33,0 & $(12-43)$ & 22,2 & $(12,2)$ & 21,0 & $(10-49)$ & 0,037 \\
\hline $\begin{array}{l}\text { WHOOQOL-BREF } \\
\text { Total }\end{array}$ & 63,3 & $(16,9)$ & 61,9 & $(39,2-96,4)$ & 68,3 & $(16,2)$ & 70,0 & $(33,9-94,6)$ & 0,285 \\
\hline Domínio Físico & 61,1 & $(17,9)$ & 60,7 & $(28,6-92,9)$ & 68,6 & $(16,3)$ & 73,2 & $(35,7-92,9)$ & 0,092 \\
\hline Domínio Psicológico & 58,7 & $(19,5)$ & 58,3 & $(25-95,8)$ & 67,5 & $(12,7)$ & 68,7 & $(45,8-91,7)$ & 0,017 \\
\hline Domínio Social & 65,0 & $(21,1)$ & 58,3 & $(41,7-100,0)$ & 66,7 & $(23,6)$ & 66,7 & $(16,7-100,0)$ & 0,620 \\
\hline Domínio Ambiental & 68,4 & $(15,0)$ & 65,6 & $(53,1-96,9)$ & 70,3 & $(17,6)$ & 71,9 & $(37,5-93,8)$ & 0,593 \\
\hline
\end{tabular}

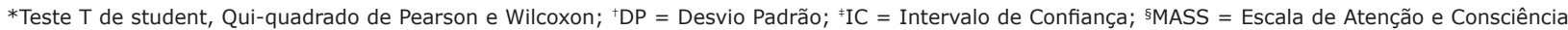
Plena; "ISS = Escala de Estresse Percebido; "WHOOQOL-BREF = Instrumento abreviado de Avaliação da Qualidade de Vida 


\section{Discussão}

A literatura tem apontado que estudantes vem apresentando altos índices de estresse ${ }^{(45-46)}$. A significativa redução do nível médio de estresse basal no presente estudo, de uma pontuação de 28,8 em T0, para 22,2 pontos em T1, com um nível de significância de $p=0,037$, evidencia a efetividade do programa. A redução de estresse é um dos pilares dos programas baseados em Mindfulness, em especial no programa utilizado como referencia para a intervenção testada no presente estudo (MBSR). Por meio da realização de práticas de Mindfulness é possível desenvolver habilidades para lidar assertivamente com eventos estressantes, diminuindo problemas de ansiedade ou outros padrões de pensamentos negativos, que podem ser o início de um ciclo de reatividade ao estresse, que contribui para o aumento de desordens psicológicas ${ }^{(47-49)}$.

Altos níveis de estresse parecem estar relacionados com efeitos deletérios ao bem-estar físico e mental e consequentemente promovem a diminuição da QV em estudantes(50-53). Após o programa de Mindfulness (T1), percebemos um aumento do nível global desse parâmetro. Também houve aumento em todos os quatro domínios separadamente quando comparados aos resultados obtidos na linha basal (TO). Destacamos como significativo o aumento do nível de QV em domínio psicológico da escala, de 58,7 em T0 para 67,5 em T1 $(p=0,017)$.

O conceito de QV é definido como a percepção do indivíduo do seu contexto cultural, sistema de valores, objetivos, expectativas, padrões e preocupações de vida(20). O estado de Mindfulness é entendido como estar consciente para o que está acontecendo no momento, relacionando-se com todas as experiências, sejam elas desagradáveis, agradáveis ou neutras, diminuindo o sofrimento e aumentando a sensação de bem estar ${ }^{(23,54)}$. Ao lidar de forma consciente e não julgadora para as situações e preocupações de vida, diminui-se o estresse e consequentemente promove-se o aumento de $\mathrm{QV}^{(23,55-}$ 56), o que ratifica os resultados desse estudo.

A Atenção Plena é caracterizada pela regulação de forma intencional da atenção ao que está acontecendo no exato momento, sendo o oposto do estado de desatenção(23), baixos níveis de AP parecem estar associados com um maior nível de estresse e maior nível de procrastinação em estudantes ${ }^{(57)}$.

Observou-se no presente estudo um aumento significativo do nível de AP nos estudantes após o programa de oito semanas baseado em Mindfulness, com aumento de 45,8 em T0 para 57,4 pontos em T1 $(p=0,005)$, avaliado por meio da escala MAAS. O programa MBSR oferece um treinamento da $A P$, promovendo maior conscientização sobre o que está acontecendo a cada momento, com uma atitude de não julgamento e aceitação da experiência. $O$ aumento da AP pode dar origem a novas formas de responder e lidar em relação a si mesmo e ao mundo(56), permitindo ao praticante relacionar-se mais diretamente com suas experiências de vida e menos com as emoções desencadeadas por essas experiências.

Uma vez que altos níveis de estresse parecem estar relacionados com baixos níveis de AP, intervenções baseadas em Mindfulness parecem estar associadas a maior resiliência ao estresse dentro do contexto universitário resultando em um aumento da AP dos estudantes, consequentemente, melhorando seu rendimento acadêmico(58).

As limitações desse estudo piloto estão caracterizadas, primariamente, por se tratar de uma amostra por conveniência, não probabilística, além de ter sido realizado em uma única instituição de ensino superior. Assim, não se trata de uma amostra completamente representativa. Porém, apesar do número restrito de sujeitos que participaram, os resultados obtidos foram consistentes e concordantes com estudos previamente realizados em populações semelhantes $^{(14,33,57,59)}$ e confirmam os benefícios das práticas de Mindfulness, onde foram demonstrados efeitos positivos nos sintomas de EP, na QV e na AP.

Os valores pré e pós intervenção mostraram diferença estatística significante, concluindo assim que esse programa de intervenção baseado em Mindfulness pode ser uma estratégia eficaz para a promoção da saúde mental dos estudantes de graduação e pósgraduação em enfermagem, no contexto brasileiro.

Vale destacar que este estudo piloto é o primeiro estudo de intervenção com um programa de Mindfulness realizado em uma população de estudantes de enfermagem no Brasil. A presença de consistência nos efeitos da intervenção, com base nos resultados deste estudo são considerados verossímeis.

\section{Conclusão}

Os resultados encontrados nesse estudo endossam que um programa de intervenção baseado em Mindfulness visando a Redução de Estresse e Aumento da Qualidade de Vida mostrou-se eficiente na redução do EP, aumento de QV e aumento do nível de AP em estudantes de graduação e pós-graduação em enfermagem.

Nesse sentido, as instituições de ensino podem considerar os resultados deste estudo como indicador da 
potencialidade de programas de intervenção baseados em Mindfulness, entre alunos de enfermagem, como uma estratégia para reduzir os níveis de estresse, aumentar a qualidade de vida e aumentar os níveis de atenção plena, com vistas a promover a saúde mental e o bem estar dos estudantes, e consequentemente, melhorando o desempenho acadêmico.

\section{Referências}

1. Banks J, Smyth E. 'Your whole life depends on it': academic stress and high-stakes testing in Ireland. J Youth Stud. 2015;18(5):598616. doi: 10.1080/13676261.2014.992317

2. Shin JC, Jung J. Academics job satisfaction and job stress across countries in the changing academic environments. Higher Educ. 2014;67(5):603-20. doi: 10.1007/s10734-013-9668-y

3. Coelho AT, Lorenzini LM, Suda EY, Rossini S, Reimão R. Sleep quality, depression and anxiety in college. Neurobiologia. [Internet]. 2010;73(1):35 - 9. [cited Dec 1 2018]. Avaliable from: <https://revistaneurobiologia. com.br/site/files/17/Volume\%2077\%20-\%20Ano\%20 2014\%20JanJun/41/5_Artigo_Pereira\%20et\%20al..pdf> 4. Lameu JN, Salazar TL, Souza WF. Prevalence of stress symptoms among students of a public university. Psicol Educ. 2016;(42):13-22. doi: https://dx.doi. org/10.5935/2175-3520.20150021

5. Moreira DP, Furegato ARF. Stress and depression among students of the last semester in two nursing courses. Rev. Latino-Am. Enfermagem. 2013;21(spe):155-62. doi: $10.1590 / S 0104-11692013000700020$

6. Mota NIF, Alves ERP, Leite GDO, Sousa BSMA, Ferreira FMO, Dias MD. Stress among nursing students at a public university. SMAD, Rev Eletrônica Saúde Mental Álcool Drog. 2016;12(3):163-70. doi: https://doi. org/10.11606/issn.1806-6976.v12i3p163-170

7. Pacheco AE, Vilela SC, Carlos ALS. Síndrome de burnout e estresse em graduandos de enfermagem. Rev Enferm Centro-Oeste Mineiro. 2014;3(3):780-7. doi: http://dx.doi.org/10.19175/recom.v0i0.415

8. Malagris LEN, Suassuna ATR, Bezerra DV, Hirata HP, Monteiro JLF, Silva LR et al. Níveis de estresse e características sociobiográficas de alunos de pósgraduação. Psicol Rev. 2009;15(2):184-203.doi: https://doi.org/10.5752/P.1678-9563.2009v15n2p184 9. Faro A. Stress and stressors in graduate programs: a study with graduate students in Brazil. Psicologia: Teor Pesq. 2013;29(1):51-60. doi: https://doi.org/10.1590/ S0102-37722013000100007
10. Selye H. Stress, a tensão da vida. $2^{a}$ ed. São Paulo: Ibrasa; 2018. 416 p.

11. Wilson MA, Grillo CA, Fadel JR, Reagan LP. Stress as a one-armed bandit: differential effects of stress paradigms on the morphology, neurochemistry and behavior in the rodent amygdala. Neurobiol Stress. 2015;1(spe.):195-208. doi: https://doi.org/10.1016/j. ynstr.2015.06.001

12. Faro A, Pereira EM. Estresse: revisão narrativa da evolução conceitual, perspetivas teóricas e metodológicas. Psicol Saúde Doenças [Internet]. 2018; 19(1):11-7. [cited 2018 Dec 19]. Avaliable from: http://www.scielo.mec.pt/scielo.php?script=sci_ arttext\&pid=S1645-00862018000100003\&lng=pt.

13. Hammen C. Stress and depression. Annu Rev Clin Psychol. 2005;1(1):293-319. doi: https://doi. org/10.1146/annurev.clinpsy.1.102803.143938

14. Kang YS, Choi SY, Ryu E. The effectiveness of a stress coping program based on mindfulness meditation on the stress, anxiety, and depression experienced by nursing students in Korea. Nurse Educ Today. 2009;29(5):53843. doi: https://doi.org/10.1016/j.nedt.2008.12.003

15. Cohen S, Kamarck T, Mermelstein R. A global measure of perceived stress. J Health Soc Behav. [Internet]. 1983;24(4):385-96. [cited 2018 Dec 19]. Avaliable from: https://pdfs.semanticscholar.org/bed9/ 2e978f5bca851a79b16d8499b8ca21eeb3d6.pdf

16. Oliveira BMD, Mininel VA, Felli VEA. Qualidade de vida de graduandos de enfermagem. Rev Bras Enferm. 2011;64(1):130-5. doi: http://dx.doi.org/10.1590/ S0034-71672011000100019.

17. Bampi LNS, Caraldi S, Guilhem D, Pompeu RB, Campos ACO. Percepção sobre qualidade de vida de estudantes de graduação em enfermagem. Rev Gaúch Enferm. 2013;34(1):125-32. doi: http://dx.doi. org/10.1590/S1983-14472013000200016

18. Botti NCL, Lima AFD, Simões WMB. Uso de substâncias psicoativas entre acadêmicos de enfermagem da Universidade Católica de Minas Gerais. SMAD, Rev. Eletrônica Saúde Mental Álcool Drog. 2010;6(1):1-13. doi: https://doi.org/10.11606/ issn.1806-6976.v6i1p1-20

19. García LH, Gherardi-Donato ECS, Castillo MMA, Silva RC, García PG, García VM. Estrés académico y consumo de alcohol en universitarios de nuevo ingreso. J Health NPEPS. [Internet]. 2017;2(1):133-47. [Acceso 18 dic 2019]. Disponible en: https://periodicos.unemat.br/ index.php/jhnpeps/article/view/1615

20. The WHOQOL Group. The development of the World Health Organization quality of life assessment instrument 
(the WHOQOL). Soc Sci Med. 1998;46(12):1569-85. doi: https://doi.org/10.1016/S0277-9536(98)00009-4

21. Bamber MD, Schneider JK. Mindfulness-based meditation to decrease stress and anxiety in college students: a narrative synthesis of the research. Rev Educ Res. 2016; 18(esp.):1-32. doi: https://doi. org/10.1016/j.edurev.2015.12.004

22. Regehr C, Glancy D, Pitts A. Interventions to reduce stress in university students: a review and meta-analysis. J Affect Disord. 2013;148(1):1-11. doi: 10.1016/j.jad.2012.11.026

23. Kabat-Zinn J. Mindfulness-based interventions in context: past, present, and future. Clin Psychol. (New York). 2003;10(2):144-56. doi: https://doi. org/10.1093/clipsy/bpg016

24. Weinstein N, Brown KW, Ryan RM. A multi-method examination of the effects of mindfulness on stress attribution, coping, and emotional well-being. J Res Pers. 2009;43(3):374-85. doi: https://doi.org/10.1016/j. jrp. 2008.12.008

25. Treadway MT, Lazar SW. Meditation and neuroplasticity: Using mindfulness to change the brain. In: Baer RA, editor. Assessing mindfulness and acceptance processes in clients: illuminating the theory and practice of change. Oakland: Context Press/New Harbinger Publications; 2010. p. 186-205.

26. Kiken LG, Garland EL, Bluth K, Palsson OS, Gaylord SA. From a state to a trait: trajectories of state mindfulness in meditation during intervention predict changes in trait mindfulness. Pers Individ Dif. 2015;81(esp.):41-6. doi: https://doi.org/10.1016/j. paid.2014.12.044

27. Baer RA, Smith GT, Hopkins J, Krietemeyer J, Toney L. Using self-report assessment methods to explore facets of mindfulness. Assessment. 2006;13(1):27-45. doi: 10.1177/1073191105283504

28. Brown KW, Ryan RM. The benefits of being present: mindfulness and its role in psychological well-being. J Pers Soc Psychol. 2003;84(4):822-48. doi: https://doi. org/10.1037/0022-3514.84.4.822

29. Barbosa P, Raymond G, Zlotnick C, Wilk J, Toomey $\mathrm{R}$, Mitchell J. Mindfulness-based stress reduction training is associated with greater empathy and reduced anxiety for graduate healthcare students. J Health Educ. 2013;26(1):9-14. doi: https://doi. org/10.4103/1357-6283.112794

30. Vibe M, Solhaug, I, Tyssen R, Friborg O, Rosenvinge $\mathrm{JH}$, Sørlie T, Bjørndal A. Mindfulness training for stress management: a randomised controlled study of medical and psychology students. BMC
Med Educ. 2013;13(1):107-19. doi: https://doi. org/10.1186/1472-6920-13-107

31. Hilton L, Hempel S, Ewing BA, Apaydin E, Xenakis $L$, Newberry $S$ et al. Mindfulness meditation for chronic pain: systematic review and meta-analysis. Ann Behav Med. 2017;51(2):199-213. doi: https://doi. org/10.1007/s12160-016-9844-2

32. Song $Y$, Lindquist $R$. Effects of mindfulnessbased stress reduction on depression, anxiety, stress and mindfulness in Korean nursing students. Nurse Educ Today. 2015;35(1):86-90. doi: https://doi. org/10.1016/j.nedt.2014.06.010

33. Shapiro SL, Brown KW, Thoresen C, Plante TG. The moderation of mindfulness-based stress reduction effects by trait mindfulness: results from a randomized controlled trial. J Health Psychol. 2011;67(3):267-77. doi: https://doi.org/10.1002/jclp.20761

34. Demarzo MMP, Andreoni S, Sanches N, Perez S, Fortes S, Garcia-Campayo J. Mindfulness-based stress reduction (MBSR) in perceived stress and quality of life: an open, uncontrolled study in a Brazilian healthy sample. Explore. (NY). 2014;10(2):118-20. doi: https:// doi.org/10.1016/j.explore.2013.12.005

35. Hirayama MS, Milani D, Rodrigues RCM, Barros NF, Alexandre NMC. A percepção de comportamentos relacionados à atenção plena e a versão brasileira do Freiburg Mindfulness Inventory. Ciênc Saúde Coletiva. 2014;19(9):3899-914. doi: https://doi. org/10.1590/1413-81232014199.12272013

36. Limberger J, Mello LTN, Schneider JA, Andretta I. Mindfulness no treinamento em habilidades sociais para usuários de drogas. Rev Bras Ter Cogn. 2017;13(2):8491. doi: http://dx.doi.org/10.5935/1808-5687.20170013 37. Carpena MX, Menezes CB. Efeito da meditação focada no estresse e mindfulness disposicional em universitários. Psicologia Teor Pesq. 2018;34(esp.):344172. doi: https://dx.doi.org/10.1590/0102.3772e3441 38. Oliveira AVRR, Padovani RC. Mindfulness e o esporte competitivo: a importância da atenção plena para atletas de alto rendimento. Rev Bras Psicol Esporte. [Internet]. 2018;8(2):42-51. [Acesso 19 dez 2018]. Disponível em: <https://bdtd.ucb.br/index.php/RBPE/article/ view/9677>

39. Gazzaz ZJ, Baig M, Al Alhendi B, Al Suliman M, Al Alhendi AS. Al-Grad M et al. Perceived stress, reasons for and sources of stress among medical students at Rabigh Medical College, King Abdulaziz University, Jeddah, Saudi Arabia. BMC Med Educ. 2018;18(1):29. doi: 10.1186/ s12909-018-1133-2

40. Moutinho ILD, Maddalena NCP, Roland RK, Lucchetti ALG, Tibiriçá SHC, Ezequiel OS et al. Depression, stress 
and anxiety in medical students: a cross-sectional comparison between students from different semesters. Rev Assoc Méd Bras. 2017;63(1):21-8. doi: https:// dx.doi.org/10.1590/1806-9282.63.01.21

41. Turner K, McCarthy VL. Stress and anxiety among nursing students: a review of intervention strategies in literature between 2009 and 2015. Nurse Educ Pract. 2017;22(1): 21-9. doi: 10.1016/j.nepr.2016.11.002 42. Barros VV. Evidências de validade da Escala de Atenção e Consciência Plenas (MAAS) e do Questionário das Facetas de Mindfulness (FFMQ-BR) entre usuários de tabaco e população geral. [Dissertação]. Juiz de Fora: Universidade Federal de Juiz de Fora; 2013. 130 p.

43. Luft CDB, Sanches SO, Mazo GZ, Andrade A. Brazilian version of the Perceived Stress Scale: translation and validation for the elderly. Rev Saúde Pública. 2007;41(4):606-15. doi: https://doi.org/ S0034-89102007000400015

44. Fleck MP, Louzada S, Xavier M, Chachamovich E, Vieira G, Santos L, Pinzon V. Application of the Portuguese version of the abbreviated instrument of quality life WHOQOL-bref. Rev Saúde Pública. 2000;34(2):178-83. doi: https://doi.org/S0034-89102000000200012.

45. Luft CDB, Sanches SO, Mazo GZ, Andrade A. Brazilian version of the Perceived Stress Scale: translation and validation for the elderly. Rev Saúde Pública. 2007;41(4):606-15. doi: https://doi.org/ S0034-89102007000400015

46. Andrews B, Wilding JM. The relation of depression and anxiety to life $\square$ stress and achievement in students. Br J Health Psychol. 2004;95(4):509-21. doi: 10.1348/0007126042369802

47. Newbury-Birch D, Lowry RJ, Kamali F. The changing patterns of drinking, illicit drug use, stress, anxiety and depression in dental students in a UK dental school: a longitudinal study. Br Dent J. 2002;192:646-9. doi: 10.1038/sj.bdj.4801448

48. Hölzel BK, Carmody J, Vangel M, Congleton C, Yerramsetti SM, Gard T, et al. Mindfulness practice leads to increases in regional brain gray matter density. Psychiatry Res. 2010;191(1):36-43. doi: 10.1016/j. pscychresns.2010.08.006

49. Teasdale JD, Segal Z, Williams J. How does cognitive therapy prevent depressive relapse and why should attentional control (mindfulness) training help? Behav Res Ther. 1995;33(1): 25-39. doi: https://doi. org/10.1016/0005-7967(94)E0011-7

50. Vibe MF, Bjørndal A, Fattah S, Dyrdal GM, Halland E, Tanner-Smith EE. Mindfulness-based stress reduction (MBSR) for improving health, quality of life and social functioning in adults: a systematic review and meta-analysis. Campbell Systematic Rev. 2017;13(11):1-127. doi: 10.4073/csr.2017.11

51. Beiter R, Nash R, McCrady M, Rhoades D, Linscomb M, Clarahan M, Sammut S. The prevalence and correlates of depression, anxiety, and stress in a sample of college students. J Affect Disord. 2015;1(173):90-6. doi: $10.1016 /$ j.jad.2014.10.054

52. Nyklíček I, Kuijpers KF. Effects of mindfulness-based stress reduction intervention on psychological well-being and quality of life: is increased mindfulness indeed the mechanism?. Ann Behav Med. 2008;35(3):331-34. doi: 10.1007/s12160-008-9030-2

53. Pacheco JP, Giacomin HT, Tam WW, Ribeiro TB, Arab C, Bezerra IM, et al. Commun Mental health problems among medical students in Brazil: a systematic review and meta-analysis. Rev Bras Psiquiatr. 2017;39(4):369-78. doi: http://dx.doi. org/10.1590/1516-4446-2017-2223

54. Germer CK, Siegel RD, Fulton PR. Mindfulness and psychotherapy. 2 ed. New York: Guilford Press; 2013. $382 \mathrm{p}$.

55. Frias CM, Whyne E. Stress on health-related quality of life in older adults: the protective nature of mindfulness. J Ment Health Aging. (Lond). 2015;19(3):201-6. doi: $10.1080 / 13607863.2014 .924090$

56. Vibe M, Hammerstrøm K, Kowalski K, Bjørndal A. Mindfulness Based Stress Reduction (MBSR) for improving health and social functioning in adults. Campbell Systematic Rev. 2017;13(11):1-15. doi: 10.4073/csr.2012.3

57. Sirois FM, Tosti N. Lost in the moment? an investigation of procrastination, mindfulness, and wellbeing. J Ration Emot Cogn Behav Ther. 2012;30(4):23748. doi: http://dx.doi.org/10.1007/s10942-012-0151-y 58. Galante J, Dufour G, Vainre M, Wagner AP, Stochl J, Benton $A$ et al. A mindfulness-based intervention to increase resilience to stress in university students (the mindful student study): a pragmatic randomised controlled trial. Lancet Public Health. 2018;3(2):72-81. doi: https://doi.org/10.1016/S2468-2667(17)30231-1

59. Beddoe $A E$, Murphy SO. Does mindfulness decrease stress and foster empathy among nursing students? J Nurs Educ. 2004;43(7):305-12. doi: https://doi. org/10.3928/01484834-20040701-07

\section{Contribuição dos autores}

Concepção e planejamento do estudo: Edilaine Cristina da Silva Gherardi-Donato, Driele Cristina Gomes Quinhoneiro e Larissa Bessani Hidalgo Gimenez. Obtenção dos dados: Driele Cristina Gomes 
Quinhoneiro e Larissa Bessani Hidalgo Gimenez. Análise e interpretação dos dados: Edilaine Cristina da Silva Gherardi-Donato, Driele Cristina Gomes Quinhoneiro, Larissa Bessani Hidalgo Gimenez, Leonardo Hernandes Siqueira, Kranya Victoria Diaz-Serrano e Ana Carolina Guidorizzi Zanetti. Análise estatística: Ana Carolina Guidorizzi Zanetti. Redação do manuscrito: Edilaine Cristina da Silva Gherardi-Donato, Driele Cristina Gomes Quinhoneiro, Larissa Bessani Hidalgo Gimenez, Leonardo Hernandes Siqueira e Kranya Victoria Diaz-Serrano. Revisão crítica do manuscrito: Edilaine Cristina da Silva Gherardi-Donato, Larissa Bessani Hidalgo Gimenez, Leonardo Hernandes Siqueira, Kranya Victoria DiazSerrano e Ana Carolina Guidorizzi Zanetti.

Todos os autores aprovaram a versão final do texto.

Conflito de interesse: os autores declararam que não há conflito de interesse. 